\title{
Thymoquinone ameliorates delayed cerebral injury and cerebral vasospasm secondary to experimental subarachnoid haemorrhage
}

\author{
Enes Akkaya ${ }^{1}$, Şevket Evran ${ }^{2}$, Fatih Çaliş ${ }^{3}$, Serdar Çevik ${ }^{4,5}$, Salim Katar ${ }^{6}$, Ersin Karataş ${ }^{7}$, \\ Abdurrahim Koçyiğit ${ }^{7}$, Mustafa Yasin Sağlam ${ }^{8}$, Mustafa Aziz Hatiboğlu ${ }^{7}$, Hakan Hanimoğlu', \\ Mehmet Yaşar Kaynar ${ }^{10}$ \\ ${ }^{1}$ Neurosurgery Department of Şişli Hamidiye Etfal Training and Research Hospital, Istanbul, Turkey \\ ${ }^{2}$ Department of Neurosurgery, Haseki Training and Research Hospital, Istanbul, Turkey \\ ${ }^{3}$ Department of Neurosurgery, Medeniyet University, Goztepe Training and Research Hospital, Istanbul, Turkey \\ ${ }^{4}$ Department of Neurosurgery, Memorial Şişli Hospital, Istanbul, Turkey \\ ${ }^{5}$ Department of Physical Therapy and Rehabilitation, School of Health Sciences, Gelisim Universty, Istanbul, Turkey \\ ${ }^{6}$ Department of Neurosurgery, Faculty of Medicine, Balikesir University, Balikesir, Turkey \\ ${ }^{7}$ Bezmialem Vakif University School of Medicine, Istanbul, Turkey \\ ${ }^{8}$ Department of Pathology, Kartal Dr. Lütfi Kirdar Education and Training Hospital, Istanbul, Turkey \\ ${ }^{9}$ Biruni University Hospital, Istanbul, Turkey \\ ${ }^{10}$ Department of Neurosurgery, Cerrahpasa Faculty of Medicine, Istanbul, Turkey
}

\begin{abstract}
Aim of the study. Among subarachnoid haemorrhage (SAH) patients, delayed cerebral injury (DCI) and infarction are the most important causes of death and major disability. Cerebral vasospasm (CVS) and DCI remain the major cause of death and disability. Thymoquinone (TQ) is the substance most responsible for the biological activity of nigella sativa (NS) and is useful in the treatment of ischaemic and neurodegenerative diseases, oxidative stress, inflammatory events, cardiovascular and neurological diseases.

We conducted an experimental study aimed to investigate the preventive and corrective effects of TQ.

Materials and methods. 24 Sprague-Dawley rats were randomly divided into three groups. The first was the control group which was a sham surgery group. The second group was the SAH group where the double haemorrage SAH protocol was used to induce vasospasm. The third group was the $\mathrm{SAH}+\mathrm{TQ}$ group, where cVS was induced by the $\mathrm{SAH}$ protocol and the animals received oral $2 \mathrm{cc}$ thymoquinone solution for seven days at a dose of $10 \mathrm{mg} / \mathrm{kg}$, after the induction of SAH. The rats were euthanised seven days after the first procedure. The degree of cerebral vasospasm was evaluated by measuring the basilar artery luminal area and arterial wall thickness. Apoptosis was measured by the western blot method at brainstem neural tissue. Oxidative stress was measured by the Erel Method. Endothelin-1 was measured with ELISA analysis at blood. Statistical analysis was performed.

Results. Endothelin-1 values were found to be statistically significantly lower in the control and $\mathrm{SAH}+\mathrm{TQ}$ groups compared to the SAH group $(\mathrm{P}<0.001)$. Mean lumen area values were significantly higher in the control and $\mathrm{SAH}+\mathrm{TQ}$ groups than in the SAH group $(P<0.001)$. In the control and $S A H+T Q$ groups, wall thickness values decreased significantly compared to the $S A H$ group $(P<0.001)$. OSI values were significantly lower in the control and $S A H+T Q$ groups than in the $S A H$ group $(P<0.001)$. Apoptosis was significantly lower in the control and $\mathrm{SAH}+\mathrm{TQ}$ groups than in the SAH group $(\mathrm{P}<0.001)$.

Conclusion. Our results show that post-SAH TQ inhibits/improves DCl and cVS with positive effects on oxidative stress, apoptosis, ET-1, lumen area, and vessel wall thickness, probably due to its anti-ischaemic, antispasmodic, antioxidant, anti-inflammatory, anti-apoptotic and neuroprotective effects.
\end{abstract}

Key words: aneurysm, thymoquinone, oxidative stress, subarachnoid haemorrhage, cerebral vasospasm, delayed cerebral injury (Neurol Neurochir Pol 2020; 54 (6): 576-584)

Address for correspondence: Enes Akkaya, Neurosurgery Department of Şişli Hamidiye Etfal Training and Research Hospital, Istanbul, Turkey, e-mail: drenesnrs@gmail.com 


\section{Introduction}

Among subarachnoid haemorrhage (SAH) patients, delayed cerebral injury (DCI) and infarction are the most important causes of death and major disability [1]. The major cause of DCI is cerebral vasospasm (cVS) [1,2]. The prevention and treatment of DCI due to vasospasm developing after SAH have been key targets. Despite surgical and endovascular developments over the last 30 years, and improved intensive care unit (ICU) support, cVS and secondary DCI remain the major cause of death and disability [3]. Because cVS can develop due to many reasons and has a complex mechanism, many studies regarding its treatment have not achieved full success [4].

Nigella sativa (NS) is a plant common in Mediterranean countries and the Indian subcontinent. Studies have shown that thymoquinone (TQ) is the substance most responsible for the biological activity of this plant (30-48\%) [5]. TQ is useful in the treatment of ischaemic and neurodegenerative diseases, oxidative stress, inflammatory events, cardiovascular and neurological diseases [6-11]. TQ has attracted the interest of researchers into various diseases due to its antispasmodic, anticonvulsant, antioxidant, and anti-inflammatory effects [12-14].

We conducted an experimental study designed to investigate the preventive and corrective effects of TQ molecule with antispasmodic, antioxidant, anti-inflammatory, and neuroprotective properties in DCI and cVS, which is the most important cause of DCI.

\section{Clinical rationale for study}

As doctors, we are still often helpless when faced with patients who develop CVS and DCI after SAH. While some of these patients survive although requiring long-term treatment, many of them die. TQ is a natural molecule with proven positive effects and no noted side effects. Although the pathogenesis of cVS and DCI is still not fully understood, certain pathways and markers have been identified. In this study, we showed that TQ improves these pathways and markers. In addition, we believe it might represent a potential cure for this disease.

\section{Material and methods}

We randomly divided 24 Sprague-Dawley rats (285-370 g, adults, males) into three groups. The first was the control group $(\mathrm{n}=8)$, in which we did not induce aSAH or treatment, and only delivered oral saline by oral gavage. In this group, we punctured the cisterna magna as detailed below and slowly drained $0.1 \mathrm{~mL}$ CSF before injecting back into the cisterna magna after the induction of anaesthesia. We then delivered 2 cc saline by oral gavage for seven days.

The second group was the SAH group $(n=8)$. Here we used the SAH method to produce vasospasm, as detailed below. We then delivered $2 \mathrm{cc}$ saline by oral gavage for seven days.
The third and final group was the SAH+TQ group $(n=8)$, where we used SAH method and gave an oral $2 \mathrm{cc}$ thymoquinone (Sigma-Aldrich (St. Louis, MO, USA)) solution for seven days at a dose of $10 \mathrm{mg} / \mathrm{kg}$ to animals after the induction of $\mathrm{SAH}$. We chose this dose according to a previous study [15].

We kept the animals at a temperature of $22^{\circ} \mathrm{C}\left( \pm 2^{\circ} \mathrm{C}\right)$ and gave them a normal diet, implementing a 12-hour light/12hour dark cycle. We gave $5 \mathrm{~mL}$ of lactated ringer's to animals intraperitoneally after the procedure. They had free access to water. All the rats breathed spontaneously during the operations. We sedated animals using a combination of intramuscular administration of $60 \mathrm{mg} / \mathrm{kg}$ ketamine hydrochloride (Parke Davis, Istanbul, Turkey) and $5 \mathrm{mg} / \mathrm{kg}$ xylazine hydrochloride [Rompun 2\% solution, $50 \mathrm{cc}$ bottle (Bayer-Türk Ilaç Ltd., Istanbul, Turkey)].

We maintained animal welfare, and all processes were in accordance with the European Communities Council Directive of 24 November, 1986 (86/609/EEC) concerning the protection of animals for experimental use. All experimental procedures used in this investigation were reviewed and approved by the ethical committee of Bezmialem University.

\section{Double haemorrhage model for cVS}

We chose this method because of its high effectiveness compared to other models [16]. Firstly, we placed rats in the supine position and catheterised tail arteries for fresh arterial blood. Then we placed rats in the prone position. We made a midline nuchal incision, and dissected cutaneous and subcutaneous tissues, fascia and muscular tissues to approach to atlanto-occipital membrane. We inserted a $25-\mathrm{G}$ injector into the cisterna magna, removed $0.1 \mathrm{~mL}$ of CSF, and injected $0.1 \mathrm{~mL}$ of fresh, non-heparinised autologous arterial blood obtained from the tail artery, aseptically into the cisterna magna within a maximum of two minutes. We sealed the puncture site with glue after the blood injection. We tilted all rats, heads lowered at an angle of $30^{\circ}$, for 30 minutes to allow blood deposition among basal cisterns. We left the rats in separate cages after recovery from anaesthesia and routinely checked their vital signs. We repeated the same routine after 48 hours. We gave animals thymoquinone solution by oral gavage for seven days, but only to the SAH+TQ group. During our experiment, five rats died and were excluded. Four of these were in the SAH group and the other was in the SAH+TQ group. We introduced new rats to the groups to replace them.

\section{Euthanisation}

Seven days after the first procedure, after sedating animals, we took blood samples $\left(10 \mathrm{~cm}^{3}\right)$ from the left ventricle for biochemical examination, and euthanised rats by beheading them. After that, we removed the brainstems carefully and preserved them in a $10 \%$ formaldehyde solution for 24 hours. We divided these tissues into two parts for pathological examination and biochemical analysis. We centrifuged blood 
samples at 1,000 $\mathrm{g}$ for five minutes, and took upper clear supernatants for examination. We stored all serum samples at $-80^{\circ} \mathrm{C}$ until analysed.

\section{Histologic morphometric analysis of the basilar artery}

We evaluated the severity of cerebral vasospasm by evaluating the basilar artery luminal area and arterial wall thickness. The basilar artery sections were deparaffinised, hydrated, washed, and stained with $\mathrm{H}$ \& E Basilar arteries. $10 \mathrm{~mm}$ thick section micrographs were analysed using ImageJ version $1.45(\mathrm{NIH})$. We calculated cross-sectional areas from the perimeter of the luminal border. We measured, and averaged for each vessel, three continuous sections (i.e. midpoint of proximal, middle, distal). The staining results were evaluated at $400 \times$ magnification by two blinded investigators with no knowledge of the treatment groups. The mean \pm SEM value obtained for each artery was used as the final value for a particular vessel.

\section{Biochemical analysis}

\section{Western blot method}

Tissues obtained from animals were homogenised with metal beads in RIPA buffer $(0.5 \mathrm{M}$ Tris- $\mathrm{HCl}, \mathrm{pH} 7.4,1.5 \mathrm{M}$ $\mathrm{NaCl}, 2.5 \%$ deoxycholic acid, 10\% NP-40, 10mM EDTA). Homogenised tissues were centriged at $14,000 \mathrm{rpm}$ for $10 \mathrm{~min}$ utes at $4^{\circ} \mathrm{C}$. Supernatants were collected to use as protein samples, and stored at $-20^{\circ} \mathrm{C}$ until experiments begin. The Bradford method was used to measure protein amount of the samples [17]. Samples were loaded to SDS polyacrylamide gel of $12 \%$ to ensure $80 \mu \mathrm{g}$ in each well. Electrophoresis was performed to allow the gel $80 \mathrm{~V}$ for 20 minutes and $120 \mathrm{~V}$ for 100 minutes. After electrophoresis, gels were transferred to the PVDF (polyvinylidene fluoride) membrane using an IBlot (Thermo Fisher, Waltham, MA, USA) device. These processes were repeated for each antibody. Bax (1:1,000 dilution, NB110-55492, Novus Biologicals, Centennial, CO, USA) Bcl-2 (1:1,000 dilution NB100-92142, Novus Biologicals), Active Caspase-3 (1:1,000 dilution, NB100-56113 Novus Biologicals) and B-Actin (1:1,000 dilution, NBP1-47423 Novus Biologicals) antibodies were used in our study. Membranes were visualised with a western blotting luminol reagent (Santa Cruz Biotechnology, Santa Cruz, CA, USA) solution for chemiluminescence imaging after treatment with each antibody. Imaging was performed via the Vilber Laurmat Fx5 Fusion (Vilber, Paris, France) imaging system. Image J (Glyko, Novato, CA, USA) software was used for analysis of the obtained images in optic density.

\section{Oxidative stress analysis}

Total antioxidant status (TAS; mmol trolox equiv/L) and total oxidant status [TOS; ( $\mu \mathrm{mol} \mathrm{H} 2 \mathrm{O} 2$ equiv/L)] were determined using commercially available kits (Rel Assay
Diagnostics; Mega Tip, Gaziantep, Turkey). The oxidative stress index [OSI; OSI (AU) = TOS $(\mathrm{mmol} \mathrm{H} 2 \mathrm{O} 2$ equiv/L)/ TAS (mmol trolox equiv/L)] was calculated. Serum TAS and TOS levels were determined via the colorimetrical method using a Varioskan Flash Multimode Reader (Thermo Fisher) and measurement methods developed by Erel [18, 19].

\section{ELISA analysis}

Tissue samples were thawed, and an Enzyme-Linked ImmunoSorbent Assay (ELISA) kit was used for the quantitative measurement of endothelin-1. The ELISA was performed according to the manufacturer's instructions (Sunlong SL0252Ra, China). Optical density was read on a standard automated plate reader at $450 \mathrm{~nm}$ (Bio-Rad, iMark Microplate Absorbance Reader).

\section{Statistical analysis}

We defined quantitative variable behaviour using measures of centralisation and variation as mean \pm SD. We used a one-way analysis of variance $F$ test to show the differences in behaviour of the group means in cases where assumptions such as independence, normality, and homoscedasticity was met. We conducted Tukey post hoc analysis for multiple comparisons. We established statistical significance as $\mathrm{P}=0.05$ for all cases. A GraphPad Prism Version 6 Software Program (GraphPad Software, San Diego, CA, USA) was used to achieve statistical analyses.

\section{Results}

When the apoptotic proteins Caspase- 3 and Bax proteins were examined, they were found to be highest in the $\mathrm{SAH}$ group and lowest in the control group (Tab. 1). Caspase-3 values were significantly lower in the control group $(\mathrm{P}<0.001)$ and in the SAH+TQ group $(\mathrm{P}<0.01)$ compared to the SAH group (Fig. 1C). Bax protein was significantly lower in the control and SAH+TQ groups than in the SAH group $(\mathrm{P}<0.001)$ There was no statistically significant difference between the SAH+TQ group and the control group for both parameters $(\mathrm{P}>0.05)$ (Fig. 1A, C).

$\mathrm{Bcl}-2$, an anti-apoptotic protein, was found to be the lowest in the SAH group and the highest in the control group (Tab. 1). Bcl-2 values in the SAH+TQ and control groups were significantly higher than in the SAH group $(\mathrm{P}<0.001)$. There was no statistically significant difference between the SAH+TQ group and the control group ( $\mathrm{P}<0.05)$ (Fig. 1B).

According to our results, endothelin-1 values were found to be the lowest in the control group, and the highest in the SAH group (Tab. 1). Endothelin-1 values were found to be statistically significantly lower in the control and SAH+TQ groups compared to the SAH group $(\mathrm{P}<0.001)$. There was no statistically significant difference between the $\mathrm{SAH}+\mathrm{TQ}$ group and the control group $(\mathrm{P}>0.05)$ (Fig. 2A). 
Table 1. Mean values and standard deviations of parameters

\begin{tabular}{|c|c|c|c|c|}
\hline PARAMETER & CONTROL GP & SAH GP & TREATMENT GP & P value \\
\hline Endothelin-1 [ng/L] & $33.19 \pm 2.311$ & $74.91 \pm 2.914$ & $41.01 \pm 1.064$ & $<0.001$ \\
\hline Luminal area & $0.1388 \pm 0.002266$ & $0.0975 \pm 0.003134$ & $0.1313 \pm 0.003981$ & $<0.001$ \\
\hline Wall thickness & $0.0225 \pm 0.001637$ & $0.03375 \pm 0.001830$ & $0.02313 \pm 0.001619$ & $<0.001$ \\
\hline TAS [Trolox equivalent/L] & $1.901 \pm 0.05300$ & $0.7463 \pm 0.08512$ & $1.733 \pm 0.0375$ & $<0.001$ \\
\hline $\mathrm{TOS}\left[\mu \mathrm{mol} \mathrm{H} \mathrm{O}_{2}\right.$ Eqv./L] & $8.029 \pm 0.2033$ & $13.33 \pm 0.3248$ & $9.760 \pm 0.3439$ & $<0.001$ \\
\hline OSI [arbitrary unit] & $425.2 \pm 18.05$ & $1,969 \pm 238.7$ & $564.9 \pm 20.01$ & $<0.001$ \\
\hline $\mathrm{Bcl}-2 / \beta$-actin & $1.883 \pm 0.05135$ & $0.3591 \pm 0.04956$ & $1.558 \pm 0.1136$ & $<0.01$ \\
\hline Caspase- $3 / \beta$-actin & $0.3948 \pm 0.08323$ & $1.691 \pm 0.1912$ & $0.6946 \pm 0.1166$ & $<0.001$ \\
\hline Bax $/ \beta$-actin & $0.4173 \pm 0.06607$ & $1.864 \pm 0.1943$ & $0.7719 \pm 0.06448$ & $<0.001$ \\
\hline
\end{tabular}

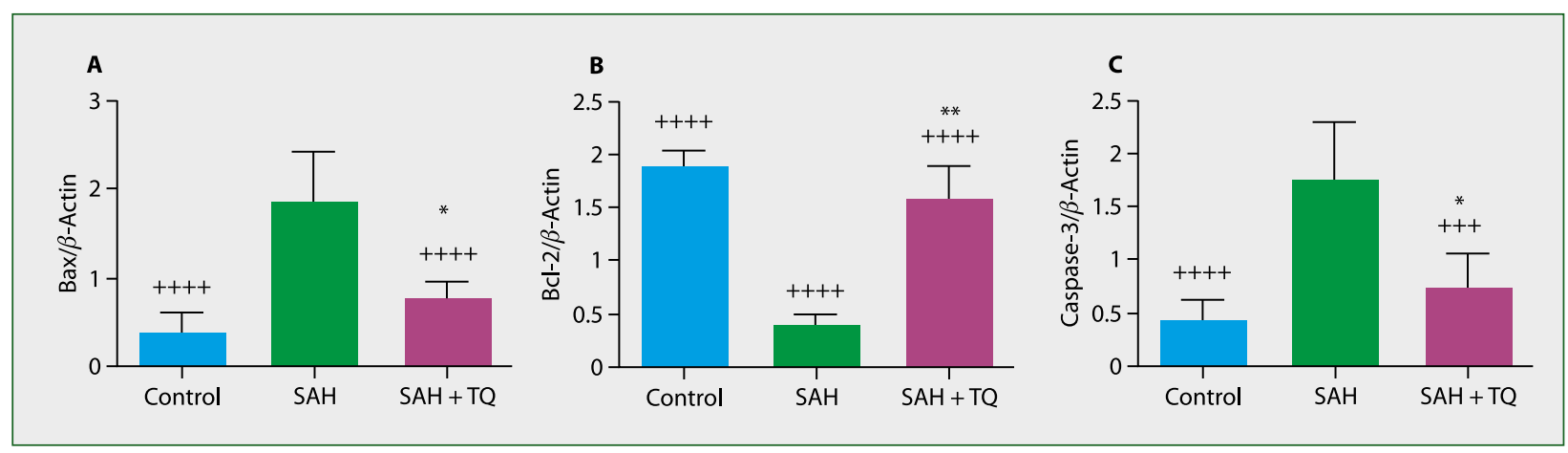

Figure 1. A. $B A X(P<0.001)$ comparing SAH group to control group and SAH+TQ group, * $P>0.05$ and comparing control group to $S A H+T Q$ group; B. BCL-2 $(\mathrm{P}<0.001)$ comparing SAH group to control group and $\mathrm{SAH}+\mathrm{TQ}$ group, $* * \mathrm{P}<0.05$ comparing control group to $\mathrm{SAH}+\mathrm{TQ}$ group; C. Caspase-3 $(\mathrm{P}<0.01)$ comparing SAH group to $\mathrm{SAH}+\mathrm{TQ}$ group, $++++\mathrm{P}<0.001$ comparing SAH group to control group, $* \mathrm{P}>0.05$ comparing control group to $\mathrm{SAH}+\mathrm{TQ}$ group

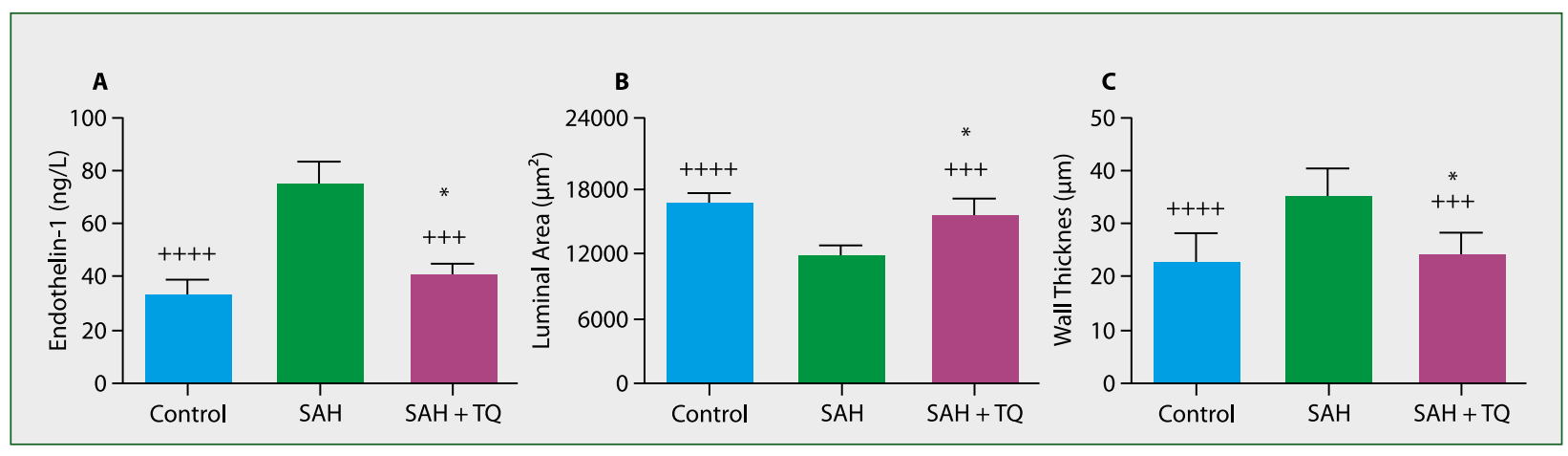

Figure 2. A. Endothelin-1 $(\mathrm{P}<0.001)$ comparing $\mathrm{SAH}$ to control and $\mathrm{SAH}+\mathrm{TQ}$ groups, ${ }^{*} \mathrm{P}>0.05$ comparing control group to $\mathrm{SAH}+\mathrm{TQ}$ group; B. Lumen area $(\mathrm{P}<0.001)$ comparing $\mathrm{SAH}$ group to control and $\mathrm{SAH}+\mathrm{TQ}$ groups, ${ }^{*} \mathrm{P}>0.05$ comparing control group to $\mathrm{SAH}+\mathrm{TQ}$ group; C. Wall thickness $(\mathrm{P}<0.001)$ comparing $\mathrm{SAH}$ to control and $\mathrm{SAH}+\mathrm{TQ}$ groups, $* \mathrm{P}>0.05$ comparing control group to $\mathrm{SAH}+\mathrm{TQ}$ group

When we look at the luminal area measurements, the luminal area which is the lowest value in the SAH group compared to the others was found to be the highest level in the control group compared to the others (Tab. 1). Mean lumen area values were significantly higher in the control and $\mathrm{SAH}+\mathrm{TQ}$ groups than in the SAH group $(\mathrm{P}<0.001)$. There was no statistically significant difference between the SAH+TQ and control groups ( $\mathrm{P}>0.05)$ (Fig. 2B).

The mean basilar artery wall thickness was the highest in the SAH group, and the lowest in the control group (Tab. 1). In the control and SAH+TQ groups, wall thickness values decreased significantly compared to the SAH group $(\mathrm{P}<0.001)$. 


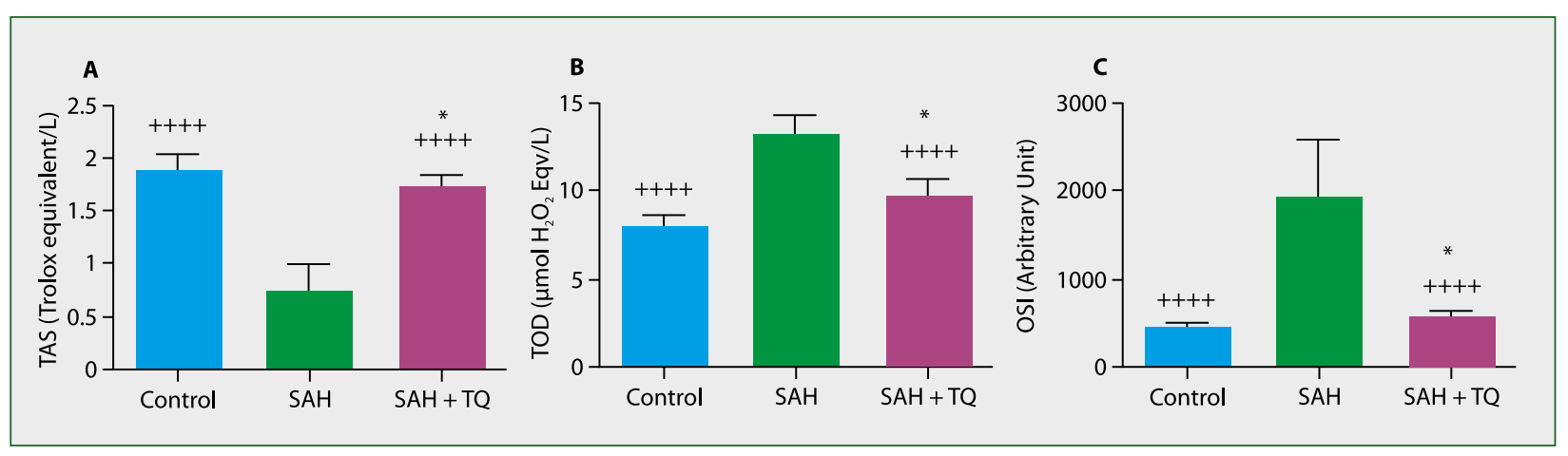

Figure 3. A. TAS $(P<0.001)$ comparing $S A H$ group to control and $S A H+T Q$ groups, $* P>0.05$ comparing control group to $S A H+T Q$ group; $\mathbf{B}$. TOS $(\mathrm{P}<0.001)$ comparing SAH group to control and SAH+TQ groups, $* * \mathrm{P}<0.05$ comparing control group to SAH+TQ group; $\mathrm{C}$. OSI ( $\mathrm{P}<$ 0.001) comparing SAH group to control and $\mathrm{SAH}+\mathrm{TQ}$ groups, $* \mathrm{P}>0.05$ comparing control group to $\mathrm{SAH}+\mathrm{TQ}$ group

There was no statistically significant difference between the SAH+TQ and control groups ( $\mathrm{P}>0.05$ ) (Fig. 2C).

The TOS and OSI values, which showed oxidative stress, reached the highest values in the SAH group compared to the other groups, and remained at the lowest levels in the control group. TAS values showing antioxidant activity were highest in the control group and lowest in the SAH group (Tab. 1). TAS values in the control and SAH+TQ groups increased significantly $(\mathrm{P}<0.001)$ compared to the SAH group, and there was no statistically significant difference between the SAH+TQ group and the control group $(\mathrm{P}>0.05)$ (Fig. 3A). TOS and OSI values were significantly lower in the control and $\mathrm{SAH}+\mathrm{TQ}$ groups than in the SAH group $(\mathrm{P}<0.001)$ (Fig. $3 \mathrm{~B}, \mathrm{C})$. There was no statistically significant difference in TOS values between the SAH+TQ and control groups $(\mathrm{P}<0.05)$ (Fig. 3B). In terms of OSI values, there was no statistically significant difference between the SAH+TQ and control groups (P > 0.05) (Fig. 3C).

\section{Discussion}

We evaluated TQ for the first time in cerebral vasospasm and delayed cerebral injury for the prevention and treatment in a rat double haemorrhage study, which is considered to be the most effective method for experimental vasospasm [16]. The results showed that the lumen area was enlarged and the wall thickness and ET-1 levels were decreased in the TQ group, which are cVS markers. This indicates that the severity of cVS decreased after TQ treatment. Also, apoptosis and oxidative stress, the markers of DCI following cVS, were significantly reduced in the TQ-treated groups. Although these positive changes in the results did not reach normal values in the control group, improvements were observed at a high level of significance.

Vasospasm-associated infarcts occur in $20-50 \%$ of patients with SAH, and severe morbidity in $30-40 \%$ [20-22]. DCI is thought to develop due to cVS and arterial contraction [23, 24]. In the chronic phase, cVS and DCI are the main culprits for poor progress. DCI is responsible for a poor outcome or death in $30 \%$ of those treated adequately after aneurysmal SAK $[25,26]$. This has been associated with many reasons, with endothelial damage, proinflammatory processes, and cVS-induced CBF reduction being the most important ones [27]. To the best of our knowledge, TQ has positive effects in preventing endothelial and ischaemic damage, and in reducing inflammation [5, 28-33]. Similar to DCI, oxidative stress, inflammatory pathways, electrolyte changes, and apoptosis, which are responsible for the pathophysiology of cVS, play an important role [22,23]. Preventive and corrective effects of TQ against oxidative stress and apoptosis have been demonstrated $[5,8,34]$. According to our results, TQ prevented and regressed many factors that play a role here.

Recently, the goal of treatment in SAH patients has been to avoid DCI by preventing cVS and to improve clinical outcomes. Therapy modalities that target not only cVS but also DCI are more effective. cVS, those secondary to cVS (DCI, apoptosis, oxidative stress), and the causes of cVS (oxidative stress, ET-1, early brain injury (EBI)) should be targeted. New molecules with anti-inflammatory, antioxidant, and antiapoptotic properties are now in the spotlight, and studies with these molecules are promising [23]. TQ also seems to be a potential treatment modality because of these features.

TQ is the most efficient ingredient/molecule of nigella sativa (NS, black seed) in essential oil; most of the beneficial properties of NS are attributable to this substance. Cell culture studies and animal models have demonstrated many therapeutic potentials of black seed and its active component, TQ, such as anti-inflammatory and antioxidant [11]. In some studies, TQ has shown the potential to improve the condition in many different types of autoimmune diseases [5]. The use of NS has been reported to be safe, with no adverse effects even at treatment doses $[9,11]$. Anti-ischaemic properties [8] and the effects of improving endothelial dysfunction $[7,28]$ are important in preventing DCI and cVS developing after SAH. This is because endothelial dysfunction progresses to vasospasm, which causes ischaemia, and ischaemia also leads to DCI. TQ, 


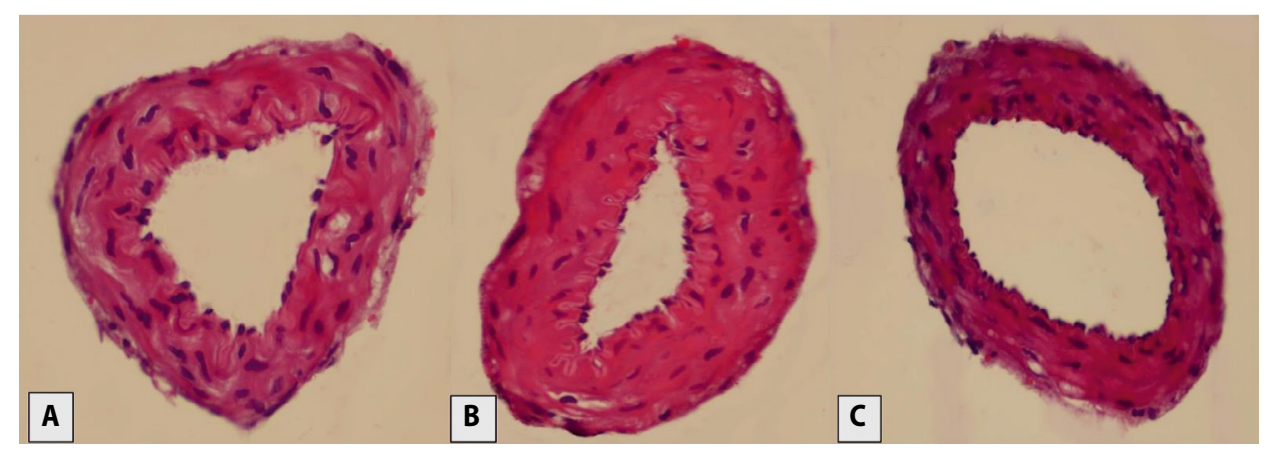

Figure 4. Cross-sectional microscopic image of basilar artery of control group (A), SAH group (B), and SAH+TQ group (C) at 400× magnification

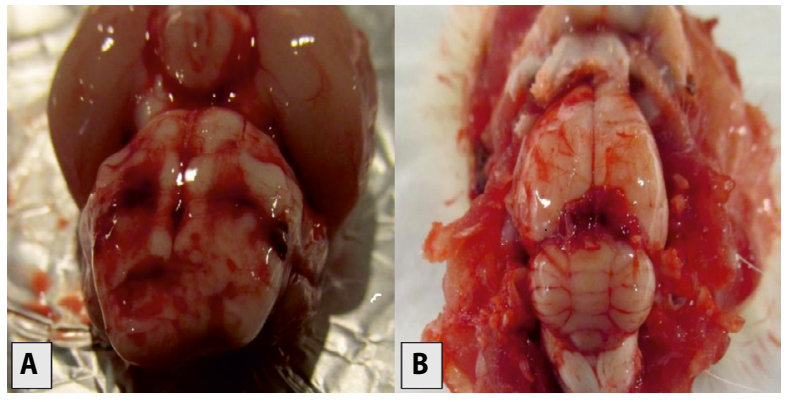

Figure 5. Basal cisterns (A) and cisterna magna with blood clots (B)

because of its antioxidant and neuroprotective properties, has the potential for the treatment of these pathological events involved in the pathogenesis of cVS and DCI. In a study similar to the ischaemia pattern in cVS, neuroprotective properties of NS were examined and positive effects were detected in MCA-occluded rats, and also neuroprotective properties were prominent in other studies [6,35-37]. Since ischaemia and infarcts due to cVS after SAH are caused by a similar mechanism, it is feasible that TQ will have the same effect.

Similarly, in our study, we started TQ immediately after the first haemorrhage, in other words before the development of cVS and DCI. We did not evaluate these substrates and enzymes in our study, but these effects may be the reason why oxidative stress falls in the treatment group.

Vasospasm is an elongated arterial constriction caused by the contraction of vascular smooth muscles [24]. SAH causes endothelial disruption and reduces susceptibility to vasodilation response by reducing nitric oxide and prostaglandin-I2 production from the endothelium [3]. Substances released from the endothelium govern events such as vascular tone adjustment, smooth muscle cell proliferation, and thrombosis $[1,38]$. TQ has the effect of dilating the pulmonary, aortae, mesenteric, and coronary arteries. It has been shown to relax smooth muscle cells $[7,39,40]$. The antispasmodic effect has also been shown to block calcium channels. In this respect, it is similar to calcium channel antagonists and nimodipine which is the only agent accepted in some treatment guidelines.
In our results, the luminal area was significantly increased and the wall thickness was significantly decreased in the TQ-treated group compared to the SAH group. Since TQ was given from the first day of bleeding, we can deduce from these results that TQ is a successful agent in preventing/regressing SAH-related cVS.

Endothelin-1 (ET-1) has the strongest role in vasoconstriction. ET-1 is more specific for cerebral circulation and stimulates smooth muscle cell proliferation, fibrosis, and inflammation. It is secreted from endothelial cells [24]. Endothelial alterations in ET-1 metabolism are thought to play an important role in the pathogenesis of cVS. Serum and plasma ET-1 levels increase after minutes of SAH. Therefore, ET- 1 is a reliable marker for the prognosis of cVS after SAH and a potent marker for radiological cVS monitoring [23, 41]. We also compared ET-1 levels to assess the effect of TQ on cVS; ET-1 levels were significantly decreased in the TQ group compared to the SAH group. Based on this, we think that the severity of cVS decreases due to TQ. Already the other indicators of cVS, luminal area and wall thickness, are also correlated with ET-1 results.

Oxidative stress plays a crucial role in EBI and triggers many events leading to DCI. There is also oxidative damage at the end of the DCI process. This correlates with the severity of DCI. Oxidative damage is both the cause and consequence of cVS and DCI. Immediately after SAH, reactive oxygen species (ROS) are produced from clots and the enzymes of the antioxidant defence systems are quickly depleted. Oxidative stress triggers many events such as endothelial damage and dysfunction, smooth muscle proliferation, activation of inflammation cascades, and production of locally potent vasoconstrictors. Because it starts to have an effect early on, treatment is very difficult in that period [23]. Various free radical scavengers have been tried to prevent cVS, but they have not been entirely successful [42]. In our results, when the oxidative stress index increased significantly in the SAH group, it decreased significantly with TQ administration. Accordingly, we argue that TQ prevents/reduces oxidative stress. Similar effects have previously been identified in other studies $[5,8,10,11,38]$. 
Secondary brain injury is mainly caused by apoptosis. Cell death begins with the activation of caspases within 24 hours after SAH [23, 43]. Oxidative stress and ischaemia trigger apoptosis [41]. TQ may prevent apoptosis by both reducing oxidative stress molecules and maintaining the width of the vessel lumen diameter. It is also known that TQ inhibits apoptosis directly through caspase pathways $[6,34,44]$.

We can say that TQ reduces apoptosis due to SAH, hypoperfusion and oxidation by decreasing caspase and Bax levels and increasing BCl-2 levels. We observed that when apoptosis levels were very high in the SAH group, they decreased significantly after TQ was administered. Thus, we conclude that TQ inhibits cVS and DCI which cause apoptosis and directly inhibits apoptosis. Bax values decreased in the TQ treatment group at a $\mathrm{P}<0.001$ significance level, while caspase- 3 values decreased at a $\mathrm{P}<0.01$ significance level.

It can be concluded that TQ inhibits Bax-induced apoptotic pathways more than caspase- 3 dependent pathways. Overall, we can talk about the positive effect of TQ because apoptosis values decreased significantly.

TQ also has effects that regulate blood pressure. In cases of hypertension involved in the treatment of cVS, it may have a negative effect on vasospasm treatment due to its effect of slightly reducing blood pressure. There will be deterioration of brain perfusion due to decreased blood pressure. However, due to its effects such as preventing endothelial damage and reducing vasospasm, these potentially negative effects have not been reflected in our experience. We have not used a method of measuring brain perfusion directly, but we can see indirect findings suggesting that brain perfusion is intact.

Several studies have shown that thrombosis in the subarachnoid space leads to inflammation which plays a major role in cVS [24, 41, 45]. TQ may also prevent cVS because it inhibits inflammation in many ways [5, 9-11, 30, 31]. We did not look at the parameter of inflammation, but we can assume this because it ultimately prevents cVS, and we intend to evaluate it in future studies.

Cognitive dysfunction is frequently experienced after SAH. There are many neurocognitive positive effects of TQ, but as far as we know no research has been conducted to evaluate this. Perhaps. in future animal and clinical studies, this parameter could be examined $[6,11]$.

\section{Clinical implıcatıons/future directions}

When we look at these results, it is difficult to understand the mechanism by which TQ makes the effects we have identified. However, it has well-known anti-ischaemic, antispasmodic, antioxidant, anti-inflammatory, anti-apoptotic and neuroprotective effects. Probably due to these effects, we found that the biochemical and histopathological parameters of cVS and DCI were improved.

Our results show that post-SAH TQ inhibits/improves DCI and cVS with positive effects on oxidative stress, apoptosis,
ET-1, lumen area, and vessel wall thickness. Using this molecule, we propose conducting a large group clinical study in cVS and DCI after SAH.

\section{References}

1. Borel CO, McKee A, Parra A, et al. Possible role for vascular cell proliferation in cerebral vasospasm after subarachnoid hemorrhage. Stroke. 2003; 34(2): 427-433, indexed in Pubmed: 12574555.

2. Abla AA, Wilson DA, Williamson RW, et al. The relationship between ruptured aneurysm location, subarachnoid hemorrhage clot thickness, and incidence of radiographic or symptomatic vasospasm in patients enrolled in a prospective randomized controlled trial. J Neurosurg. 2014; 120(2): 391-397, doi: 10.3171/2013.10.JNS13419, indexed in Pubmed: 24313610.

3. Baggott CD, Aagaard-Kienitz B. Cerebral vasospasm. Neurosurg Clin N Am. 2014; 25(3): 497-528, doi: 10.1016/j.nec.2014.04.008, indexed in Pubmed: 24994087.

4. Bele S, Proescholdt MA, Hochreiter A, et al. Continuous intra-arterial nimodipine infusion in patients with severe refractory cerebral vasospasm after aneurysmal subarachnoid hemorrhage: a feasibility study and outcome results. Acta Neurochir (Wien). 2015; 157(12): 2041-2050, doi: 10.1007/s00701-015-2597-z, indexed in Pubmed: 26439105.

5. Woo CC, Kumar AP, Sethi G, et al. Thymoquinone: potential cure for inflammatory disorders and cancer. Biochem Pharmacol. 2012; 83(4): 443-451, doi: 10.1016/j.bcp.2011.09.029, indexed in Pubmed: 22005518.

6. Beheshti F, Khazaei M, Hosseini M. Neuropharmacological effects of Nigella sativa. Avicenna J Phytomed. 2016; 6(1): 104-116, indexed in Pubmed: 27247928.

7. Detremmerie CMS, Leung SWS, Vanhoutte PM. Activation of NQO-1 mediates the augmented contractions of isolated arteries due to biased activity of soluble guanylyl cyclase in their smooth muscle. Naunyn Schmiedebergs Arch Pharmacol. 2018; 391(11): 1221-1235, doi: 10.1007/s00210-018-1548-7, indexed in Pubmed: 30062552.

8. Elmaci I, Altinoz MA. Thymoquinone: An edible redox-active quinone for the pharmacotherapy of neurodegenerative conditions and glial brain tumors. A short review. Biomed Pharmacother. 2016; 83: 635-640, doi: 10.1016/j.biopha.2016.07.018, indexed in Pubmed: 27459120.

9. Gholamnezhad Z, Havakhah S, Boskabady MH. Preclinical and clinical effects of Nigella sativa and its constituent, thymoquinone: A review. J Ethnopharmacol. 2016; 190: 372-386, doi: 10.1016/j. jep.2016.06.061, indexed in Pubmed: 27364039.

10. Javidi S, Razavi BM, Hosseinzadeh H. A review of Neuropharmacology Effects of Nigella sativa and Its Main Component, Thymoquinone. Phytother Res. 2016; 30(8): 1219-1229, doi: 10.1002/ptr.5634, indexed in Pubmed: 27169925.

11. Tavakkoli A, Mahdian V, Razavi BM, et al. Review on Clinical Trials of Black Seed (Nigella sativa) and Its Active Constituent, Thymoquinone. J Pharmacopuncture. 2017; 20(3): 179-193, doi: 10.3831/ KPI.2017.20.021, indexed in Pubmed: 30087794.

12. Akhondian J, Parsa A, Rakhshande H. The effect of Nigella sativa L. (black cumin seed) on intractable pediatric seizures. Med Sci Monit. 2007; 13(12): CR555-CR559, indexed in Pubmed: 18049435.

13. Akhondian J, Kianifar H, Raoofziaee M, et al. The effect of thymoquinone on intractable pediatric seizures (pilot study). Epilepsy Res. 2011; 
93(1): 39-43, doi: 10.1016/j.eplepsyres.2010.10.010, indexed in Pubmed: 21112742.

14. Raza M, Alghasham AA, Alorainy MS, et al. Potentiation of Valproate-induced Anticonvulsant Response by Nigella sativa Seed Constituents: The Role of GABA Receptors. Int J Health Sci (Qassim). 2008; 2(1): 15-25, indexed in Pubmed: 21475467.

15. Shao YY, Li B, Huang YM, et al. Thymoquinone Attenuates Brain Injury via an Anti-oxidative Pathway in a Status Epilepticus Rat Model. Transl Neurosci. 2017; 8: 9-14, doi: 10.1515/tnsci-2017-0003, indexed in Pubmed: 28400978.

16. Meguro T, Clower BR, Carpenter R, et al. Improved rat model for cerebral vasospasm studies. Neurol Res. 2001; 23(7): 761-766, doi: 10.1179/016164101101199144, indexed in Pubmed: 11680518.

17. Hildebrandt S, Steinhart $H$, Paschke A, et al. A rapid and sensitive method for the quantitation of microgram quantities of protein utilizing the principle of protein-dye binding. Anal Biochem. 1976; 72(3): 248254, doi: 10.1006/abio.1976.9999, indexed in Pubmed: 942051.

18. Erel O. A novel automated direct measurement method for total antioxidant capacity using a new generation, more stable ABTS radical cation. Clin Biochem. 2004; 37(4): 277-285, doi: 10.1016/j.clinbiochem.2003.11.015, indexed in Pubmed: 15003729.

19. Erel 0 . A new automated colorimetric method for measuring total oxidant status. Clin Biochem. 2005; 38(12): 1103-1111, doi: 10.1016/j. clinbiochem.2005.08.008, indexed in Pubmed: 16214125.

20. Weyer GW, Nolan CP, Macdonald RL. Evidence-based cerebral vasospasm management. Neurosurg Focus. 2006; 21(3): E8, doi: 10.3171/foc.2006.21.3.8, indexed in Pubmed: 17029347.

21. Ehlert A, Schmidt C, Wölfer J, et al. Molsidomine for the prevention of vasospasm-related delayed ischemic neurological deficits and delayed brain infarction and the improvement of clinical outcome after subarachnoid hemorrhage: a single-center clinical observational study. J Neurosurg. 2016; 124(1): 51-58, doi: 10.3171/2014.12.JNS13846, indexed in Pubmed: 26162034.

22. Dabus G, Nogueira RG. Current options for the management of aneurysmal subarachnoid hemorrhage-induced cerebral vasospasm: a comprehensive review of the literature. Interv Neurol. 2013; 2(1): 30-51, doi: 10.1159/000354755, indexed in Pubmed: 25187783.

23. Cossu G, Messerer M, Oddo M, et al. To look beyond vasospasm in aneurysmal subarachnoid haemorrhage. Biomed Res Int. 2014; 2014: 628597, doi: 10.1155/2014/628597, indexed in Pubmed: 24967389.

24. Findlay JM, Nisar J, Darsaut T. Cerebral Vasospasm: A Review. Can J Neurol Sci. 2016; 43(1): 15-32, doi: 10.1017/cjn.2015.288, indexed in Pubmed: 26332908.

25. Dorsch NW. A review of cerebral vasospasm in aneurysmal subarachnoid haemorrhage Part II: Management. J Clin Neurosci. 1994; 1(2): 78-92, doi: 10.1016/0967-5868(94)90080-9, indexed in Pubmed: 18638735.

26. Hijdra A, van Gijn J, Nagelkerke NJ, et al. Prediction of delayed cerebral ischemia, rebleeding, and outcome after aneurysmal subarachnoid hemorrhage. Stroke. 1988; 19(10): 1250-1256, doi: 10.1161/01. str.19.10.1250, indexed in Pubmed: 3176085.

27. Hendrix P, Foreman PM, Harrigan MR, et al. Endothelial Nitric Oxide Synthase Polymorphism Is Associated with Delayed Cerebral Ischemia Following Aneurysmal Subarachnoid Hemorrhage. World Neurosurg. 2017; 101: 514-519, doi: 10.1016/j.wneu.2017.02.062, indexed in Pubmed: 28254540.

28. Idris-Khodja N, Schini-Kerth V. Thymoquinone improves aging-related endothelial dysfunction in the rat mesenteric artery. Naunyn Schmie- debergs Arch Pharmacol. 2012; 385(7): 749-758, doi: 10.1007/ s00210-012-0749-8, indexed in Pubmed: 22526469.

29. Mohamed A, Afridi DM, Garani 0, et al. Thymoquinone inhibits the activation of NF-kappaB in the brain and spinal cord of experimental autoimmune encephalomyelitis. Biomed Sci Instrum. 2005; 41: 388-393, indexed in Pubmed: 15850137.

30. Salem ML. Immunomodulatory and therapeutic properties of the Nigella sativa L. seed. Int Immunopharmacol. 2005; 5(13-14): 17491770, doi: 10.1016/j.intimp.2005.06.008, indexed in Pubmed: 16275613.

31. Al-Ghamdi MS. The anti-inflammatory, analgesic and antipyretic activity of Nigella sativa. J Ethnopharmacol. 2001; 76(1): 45-48, doi: 10.1016/s0378-8741(01)00216-1, indexed in Pubmed: 11378280.

32. Hosseinzadeh $\mathrm{H}$, Parvardeh S, Asl MN, et al. Effect of thymoquinone and Nigella sativa seeds oil on lipid peroxidation level during global cerebral ischemia-reperfusion injury in rat hippocampus. Phytomedicine. 2007; 14(9): 621-627, doi: 10.1016/j.phymed.2006.12.005, indexed in Pubmed: 17291733.

33. Dariani S, Baluchnejadmojarad T, Roghani M. Thymoquinone attenuates astrogliosis, neurodegeneration, mossy fiber sprouting, and oxidative stress in a model of temporal lobe epilepsy. J Mol Neurosci. 2013; 51(3): 679-686, doi: 10.1007/s12031-013-0043-3, indexed in Pubmed: 23794216.

34. Alhebshi AH, Gotoh M, Suzuki I. Thymoquinone protects cultured rat primary neurons against amyloid $\beta$-induced neurotoxicity. Biochem Biophys Res Commun. 2013; 433(4): 362-367, doi: 10.1016/j. bbrc.2012.11.139, indexed in Pubmed: 23537659.

35. Sedaghat R, Roghani M, Khalili M. Neuroprotective effect of thymoquinone, the nigella sativa bioactive compound, in 6-hydroxydopamine-induced hemi-parkinsonian rat model. Iran J Pharm Res. 2014; 13(1): 227-234, indexed in Pubmed: 24734075.

36. Hobbenaghi R, Javanbakht J, Sadeghzadeh Sh, et al. Neuroprotective effects of Nigella sativa extract on cell death in hippocampal neurons following experimental global cerebral ischemia-reperfusion injury in rats. J Neurol Sci. 2014; 337(1-2): 74-79, doi: 10.1016/j. jns.2013.11.019, indexed in Pubmed: 24314720.

37. Kanter M. Nigella sativa and derived thymoquinone prevents hippocampal neurodegeneration after chronic toluene exposure in rats. Neurochem Res. 2008; 33(3): 579-588, doi: 10.1007/s11064-0079481-z, indexed in Pubmed: 17929168.

38. Ahmad A, Khan RMA, Alkharfy KM. Effects of selected bioactive natural products on the vascular endothelium. J Cardiovasc Pharmacol. 2013; 62(2): 111-121, doi: 10.1097/FJC.0b013e3182927e47, indexed in Pubmed: 23599064.

39. Suddek GM. Thymoquinone-induced relaxation of isolated rat pulmonary artery. J Ethnopharmacol. 2010; 127(2): 210-214, doi: 10.1016/j.jep.2009.11.019, indexed in Pubmed: 19961917.

40. Ghayur MN, Gilani AH, Janssen L. Intestinal, airway, and cardiovascular relaxant activities of thymoquinone. Evid Based Complement Alternat Med. 2012; 2012: 305319, doi: 10.1155/2012/305319, indexed in Pubmed: 23320027.

41. Przybycien-Szymanska MM, Ashley WW. Biomarker Discovery in Cerebral Vasospasm after Aneurysmal Subarachnoid Hemorrhage. J Stroke Cerebrovasc Dis. 2015; 24(7): 1453-1464, doi: 10.1016/j. jstrokecerebrovasdis.2015.03.047, indexed in Pubmed: 25957908.

42. Matsuda N, Ohkuma H, Naraoka M, et al. Role of oxidized LDL and lectin-like oxidized LDL receptor-1 in cerebral vasospasm after subarachnoid hemorrhage. J Neurosurg. 2014; 121(3): 621-630, doi: 10.3171/2014.5.JNS132140, indexed in Pubmed: 24949677. 
43. Cahill J, Calvert JW, Solaroglu I, et al. Vasospasm and p53-induced apoptosis in an experimental model of subarachnoid hemorrhage. Stroke. 2006; 37(7): 1868-1874, doi: 10.1161/01. STR.0000226995.27230.96, indexed in Pubmed: 16741174.

44. Ullah I, Ullah N, Naseer MI, et al. Neuroprotection with metformin and thymoquinone against ethanol-induced apoptotic neurodegeneration in prenatal rat cortical neurons. BMC Neurosci. 2012; 13: 11, doi: 10.1186/1471-2202-13-11, indexed in Pubmed: 22260211.

45. Cheng MF, Song JN, Li DD, et al. The role of rosiglitazone in the proliferation of vascular smooth muscle cells after experimental subarachnoid hemorrhage. Acta Neurochir (Wien). 2014; 156(11): 2103-2109, doi: 10.1007/s00701-014-2196-4, indexed in Pubmed: 25139403. 